\title{
Anharmonic effect of the rate constant of the reactions of CH3SCH2OO system in high-temperature combustion
}

\begin{tabular}{|c|c|}
\hline Journal: & Canadian Journal of Chemistry \\
\hline Manuscript ID & cjc-2017-0216.R1 \\
\hline Manuscript Type: & Article \\
\hline Date Submitted by the Author: & 27-May-2017 \\
\hline Complete List of Authors: & $\begin{array}{l}\text { Hao, Yu; Dalian Maritime University, Dalian } 116026, \text { China } \\
\text { Pan, Xinxiang; Dalian Maritime University, Dalian 116026, China } \\
\text { Song, Li; Marine Engineering College, Dalian Maritime University } \\
\text { Ding, Yang; Marine Engineering College, Dalian Maritime University } \\
\text { Xia, Wen wen; Dalian Maritime University, Dalian 116026, China } \\
\text { Wang, Shiye; Dalian Maritime University, Dalian 116026, China } \\
\text { Yu, Hongjing; Dalian Maritime University, Dalian 116026, China } \\
\text { Kang, Liqiang; Dalian Maritime University, Dalian 116026, China } \\
\text { Yao, Li; Dalian Maritime University, Dalian 116026, China }\end{array}$ \\
\hline $\begin{array}{l}\text { Is the invited manuscript for } \\
\text { consideration in a Special } \\
\text { Issue?: }\end{array}$ & N/A \\
\hline Keyword: & $\begin{array}{l}\text { anharmonic effect, RRKM theory, high-temperature combustion, rate } \\
\text { constant }\end{array}$ \\
\hline
\end{tabular}




\title{
Anharmonic effect of the rate constant of the reactions of $\mathrm{CH}_{3} \mathrm{SCH}_{2} \mathrm{OO}$ system in high-temperature combustion
}

Yu Hao, Xinxiang Pan, Liguo Song, Yang Ding, Wenwen Xia, Shiye Wang, Hongjing Yu, Liqiang Kang, Li Yao*

Dalian Maritime University, Dalian 116026, China

\begin{abstract}
The study mainly focuses on the anharmonic effect of the reactions of $\mathrm{CH}_{3} \mathrm{SCH}_{2} \mathrm{OO}$ system. The geometries of the reactants and the transition states are optimized with Gaussian 09. The barrier heights are calculated with the energy of the reactants and the transition states. The RRKM theory is utilized to calculate the anharmonic and harmonic rate constants of the reactions. The anharmonic effect of these reactions can be demonstrated obviously by our results. Generally speaking, in the study, for most reactions, the rate constants increase with the temperature in the canonical case and the total energy in the microcanonical case, and the anharmonic effect of these reactions is significant and should not be neglected in high-temperature combustion. In $\mathrm{CH}_{3} \mathrm{SCH}_{2} \mathrm{OO}$ system, $\mathrm{CH}_{3} \mathrm{SCH}_{2} \mathrm{OO} \rightarrow \mathrm{CH}_{2} \mathrm{SCH}_{2} \mathrm{OOH} \rightarrow \mathrm{CH}_{2} \mathrm{~S}+\mathrm{CH}_{2} \mathrm{O}+\mathrm{OH}$ is the main reaction channel. After a series of calculations, the anharmonic effect is remarkable, especially in high-temperature combustion. By analyzing other meaningful reactions which followed by that channel above, it can be acquired that the anharmonic effect of these reactions is generally obvious enough, especially for those reactions whose barrier heights are relatively low.
\end{abstract}


Keywords: anharmonic effect; rate constant; RRKM theory; hightemperature combustion

\section{Introduction}

The dimethyl sulfide $\left(\mathrm{CH}_{3} \mathrm{SCH}_{3}\right)$, which often comes from the marine environment, is likely to be transformed into its final oxidation products sulfuric acid and methanesulfonic acid after a series of reactions. ${ }^{1-3}$ Researchers have made efforts to study the atmospheric oxidation mechanism of $\mathrm{CH}_{3} \mathrm{SCH}_{3}{ }^{4}$ The significant reaction of initial oxidation of $\mathrm{CH}_{3} \mathrm{SCH}_{3}$ is that it reacts with $\mathrm{OH}$ radicals.

$$
\mathrm{CH}_{3} \mathrm{SCH}_{3}+\mathrm{OH} \rightarrow \mathrm{CH}_{3} \mathrm{SCH}_{2}+\mathrm{H}_{2} \mathrm{O}
$$

Almost at the same time, the radical $\mathrm{CH}_{3} \mathrm{SCH}_{2}$, one of the products, combines rapidly with $\mathrm{O}_{2}$ to form $\mathrm{CH}_{3} \mathrm{SCH}_{2} \mathrm{OO}$ (methyl-thiol-methyl peroxy radical). ${ }^{5}$ In the general atmosphere environment, $\mathrm{CH}_{3} \mathrm{SCH}_{2} \mathrm{OO}$ will react with $\mathrm{NO}, \mathrm{HO}_{2}$, and $\mathrm{RO}_{2}$ radicals. ${ }^{6-12}$ When the concentrations of $\mathrm{NO}, \mathrm{HO}_{2}$ and $\mathrm{RO}_{2}$ are extremely low, thus it creates the possibility to promote the intramolecular hydrogen shift in $\mathrm{CH}_{3} \mathrm{SCH}_{2} \mathrm{OO}$ radical. The shift will cause other reactions. The reaction process is roughly that, $\mathrm{CH}_{3} \mathrm{SCH}_{2} \mathrm{OO} \rightarrow \mathrm{CH}_{2} \mathrm{SCH}_{2} \mathrm{OOH} \rightarrow$ products. Jin et al. ${ }^{13}$ have done much theoretical study about these reactions. He found that $\mathrm{CH}_{3} \mathrm{SCH}_{2} \mathrm{OO} \rightarrow$ $\mathrm{CH}_{2} \mathrm{SCH}_{2} \mathrm{OOH} \rightarrow \mathrm{CH}_{2} \mathrm{~S}+\mathrm{CH}_{2} \mathrm{O}+\mathrm{OH}$ was dominative at temperatures above $1500 \mathrm{~K}$. The initial isomerization is essential by reason that it recycles $\mathrm{OH}$ radical. ${ }^{14}$ However, there are two main reasons why researchers have not shown much interest in it until now. One is that the 
initial isomerization is hard to take place at atmospheric temperatures on the basis of all of the atmospheric mechanisms. Another is that concentrations of $\mathrm{NO}$ and/or $\mathrm{HO}_{2}$ and $\mathrm{RO}_{2}$ are relatively high in previous laboratory study. H-shift is difficult to take place. Thus, there is little research have been carried out on the $\mathrm{CH}_{3} \mathrm{SCH}_{2} \mathrm{OO}$ system, and the related studies of the anharmonic effect are extremely limited. Our research focuses mainly on the anharmonic effect, especially in hightemperature combustion environment. Besides, after the two reactions, $\mathrm{CH}_{2} \mathrm{~S}$ (methane thial), as one of its products, need to be paid more attention to combine with $\mathrm{HO}_{2}$. The reason is that $\mathrm{CH}_{2} \mathrm{~S}$ is a typical sulfureted pollutant as a small molecule, and $\mathrm{HO}_{2}$ is an important active intermediate. Thus our study has also included another two possible reaction channels whose initial reactants are $\mathrm{CH}_{2} \mathrm{~S}$ and $\mathrm{HO}_{2}$. All the eight reactions ( $\mathrm{R} 1$ to $\mathrm{R} 8$ ) and their calculated barrier heights are given in Table 1. The reactions $\mathrm{R} 2$ and $\mathrm{R} 3$ are another two possible reaction channels in $\mathrm{CH}_{3} \mathrm{SCH}_{2} \mathrm{OO}$ system. The purpose of this paper is to investigate the anharmonic effect of the important reactions and other related reactions with the transition state theory (TST) and the RRKM theory. Furthermore, with the system of the TST, it is not difficult to calculate the rate constants of these reactions. It is a meaningful work which contributes to comparing the reaction rate constants of these reactions with those of other bimolecular combinations. And it also lays the theoretical foundation of exploring the reaction mechanism of sulphide in the high-temperature combustion environment. 


\section{Computational methods}

\subsection{Ab initio calculations}

For the $\mathrm{CH}_{3} \mathrm{SCH}_{2} \mathrm{OO}$ system shown in Table 1, the geometries of the reactants, products and transition states have been optimized with 6$311++\mathrm{G}^{* *}$ basis set. TS1, TS4, TS5, TS6, TS7 and TS8 were optimized at the MP2/6-311++G ${ }^{* *}$ level. However, we had difficulty in optimizing the geometries of TS2 and TS3 at the same level because of their higher barrier heights. They were optimized at the B3LYP/6-311++G** level. Furthermore, all the geometries of the transition states were verified by IRC (intrinsic reaction coordinate) calculations. The vibrational harmonic and anharmonic frequencies, which were calculated at the same level, are used for characterization of stationary points, zero-point-energy corrections, as well as for the calculations of reaction rate constant within the framework of the TST (transition state theory) and the RRKM (RiceRamsperger-Kassel-Marcus) theory, respectively. All stationary points have been positively identified as local minima or transition states. For the purpose of achieving higher accuracy, the coupled cluster CCSD (T)/6-311++G (d, p) method is employed to recalculate the single-point energies. We utilized Gaussian 09 program for all ab initio calculations.

\subsection{RRKM theory and anharmonic effect}

For a microcanonical system, according to RRKM theory, with energy $E$, the unimolecular reaction rate constant for the system is given by ${ }^{15}$ 


$$
k_{\mathrm{u}}(E)=\frac{1}{h} \cdot \frac{W^{\neq}\left(E-E_{\mathrm{a}}^{\neq}\right)}{\rho(E)}
$$

where $h$ is Planck's constant, $W^{\neq}\left(E-E_{\mathrm{a}}^{\neq}\right)$is the total amount of states for the transition states (TS), $\rho(E)$ is the density of the states of the reactant molecule, besides, $E$ and $E_{\mathrm{a}}^{\neq}$are the total energy and the activity energy in the microcanonical case, respectively.

According to the definition of $W(E)$, the expression of the total amount of state is given by ${ }^{16-18}$

$$
W(E)=\sum_{i}^{\text {states }} H\left(E-E_{i}\right)
$$

where $H\left(E-E_{i}\right)$ is the Heaviside function. When $E_{i}$ of those states are below or equal to $E$, we can easily use the direct counting method to obtain $W(E)$.

In general, the definition of $\rho(E)$ is given by

$$
\rho(E)=\frac{\mathrm{d} W(E)}{\mathrm{d} E}
$$

In order to attain value of $\mathrm{W}(E)$ and $\rho(E)$, applying the Laplace transformation and inverse Laplace transformation to $\mathrm{W}(E)$ and $\rho(E)$ is necessary enough.

$$
\int_{0}^{\infty} d E e^{-\beta E} W(E)=\frac{Q(\beta)}{\beta}=L[W(E)]
$$




$$
\begin{gathered}
W(E)=L^{-1}\left[\frac{Q(\beta)}{\beta}\right] \\
\int_{0}^{\infty} d E e^{-\beta E} \rho(E)=Q(\beta)=L[\rho(E)] \\
\rho(E)=L^{-1}[Q(\beta)]
\end{gathered}
$$

where $Q(\beta)$ is the partition function of the system, $\beta=\frac{1}{k T}$, where $k$ is Boltzmann's constant, $T$ is the temperature of the system. More details can be found in Ref $19 .^{19}$

For a canonical case, according to the transition state theory, the rate constant $k(T)$ in a certain unimolecular reaction can be given by ${ }^{16-18,20-21}$

$$
k_{h}(T)=\frac{k T}{h} \cdot \frac{Q^{\neq}(T)}{Q(T)} e^{-\frac{E_{a}^{*}}{k T}}
$$

here, $k$ and $T$ are Boltzmann's constant and the temperature in the canonical case, respectively. Moreover, $Q^{\neq}(T)$ is the partition function of the activated complex. $Q(T)$ is the partition function of the reactant. And we have

$$
Q(T)=\prod_{i}^{N} Q_{i}(T) \quad Q^{\neq}(T)=\prod_{i}^{N-1} Q_{i}^{\neq}(T)
$$


here, $N$ is the number of the vibration modes of the reactant, $Q_{i}(T)$ and $Q^{\ddagger}(T)$ are the vibration partition function for reactant and the activated complex for each mode, respectively.

For the purpose of calculating $k_{\mathrm{u}}(E)$ and $k_{h}(T)$, based on the discussion above, it is crucial to calculate the partition function initially. Then we shall apply Morse oscillator model (MO) to calculate the partition function. It is an appropriate way to investigate the anharmonic effect of these reactions in our study. Here are two reasons. One is that the Morse oscillator implicitly includes the phenomenon of the bond breading and has a good approximation with a fine structure of the molecular vibration. The other is that the Morse oscillator is an analytical solution to the Schrodinger equation. It is convenient for us to analyze the problems. In the case, the expression of the energy in $i$-th vibrational mode is given by

$$
E_{n_{i}}=\left(n_{i}+\frac{1}{2}\right) \hbar \omega_{i}-x_{i}\left(n_{i}+\frac{1}{2}\right)^{2} \hbar \omega_{i}
$$

here, $n_{i}$ and $\omega_{i}$ are the vibrational quantum number and the frequency of the $i$-th vibrational mode, respectively.

Besides, $x_{i}$ stands for MO parameter, its expression is given by

$$
x_{i}=\frac{\hbar \omega_{i}}{4 D_{i e}}
$$


here, $D_{i e}$ means the well depth of MO. In our own study, the MO parameter $x_{i}$ for different molecules can be acquired by anharmonic frequency calculations in Gaussian 09. The maximum of $n_{i}$ is given by

$$
n_{i}(\mathrm{~m})=\frac{1}{2 x_{i}}-\frac{1}{2}
$$

Compared with harmonic model, it is easy to find that the energy spacings are not equal for anharmonic model. In addition, there is a maximum quantum number $n_{i}(\mathrm{~m})$ in MO. In our study, all of the vibrational modes are treated as anharmonic Morse oscillator, the correctness of which has been confirmed. We can use Morse model to simulate all the degrees of freedom, include non-stretching and stretching bonds. Notably, only vibrational degrees of freedom are included in our study. Actually, the contribution of rotational degrees of freedom can easily be taken into account in our method for the RRKM calculation through the calculation of rotational partition function. Furthermore, we can even take into consideration the effect of vibration-rotation interaction. It will be carried out in future work. For initial reactions of $\mathrm{CH}_{3} \mathrm{SCH}_{2} \mathrm{OO}$ system, with the view of calculating the density of states $\rho(E)$ of reactants, harmonic and anharmonic vibrational degrees of freedom of the reactants are $24(3 \mathrm{~N}-6, \mathrm{~N}=10, \mathrm{~N}$ is the number of atoms included in $\mathrm{CH}_{3} \mathrm{SCH}_{2} \mathrm{OO}$ radical). To calculate the total number of states $W(E)$, the harmonic and anharmonic imaginary frequencies are excluded and the degrees of freedom of the transition states are 23. It is worth 
noting that the used model is a local mode anharmonic model and not a normal mode anharmonic model. It is different from the assumption of the RRKM theory which indicates that the energy levels are highly mixed and quantum numbers cannot be assigned to the levels. ${ }^{22}$ In the calculations for each vibrational mode, we choose the harmonic frequencies and $x_{i}$ as effective dissociation energy parameters for the Morse potential.

\section{Result and Discussion}

\subsection{The rate constants of initial reactions of $\mathrm{CH}_{3} \mathrm{SCH}_{2} \mathrm{OO}$ system}

In Table 1, for the first four unimolecular reactions (R1 to R4), after $a b$ initio calculations, the relative energies of all the transition states and products are shown in Figure 1. The energy of the reactant is set to zero $\mathrm{kcal} / \mathrm{mol}$. Compared to the reactant $\left(\mathrm{CH}_{3} \mathrm{SCH}_{2} \mathrm{OO}\right)$, the relative energy of TS1, TS2 and TS3 are 21.10, 38.80 and $40.12 \mathrm{kcal} / \mathrm{mol}$, respectively. It can be seen that relative energy of TS1 is the lowest. Besides, for the reaction $\mathrm{R} 4\left(\mathrm{CH}_{2} \mathrm{SCH}_{2} \mathrm{OOH} \rightarrow \mathrm{TS} 4 \rightarrow \mathrm{CH}_{2} \mathrm{~S}+\mathrm{CH}_{2} \mathrm{O}+\mathrm{OH}\right)$, the barrier height is low to $4.03 \mathrm{kcal} / \mathrm{mol}$. Hence, $\mathrm{CH}_{3} \mathrm{SCH}_{2} \mathrm{OO} \rightarrow \mathrm{CH}_{2} \mathrm{SCH}_{2} \mathrm{OOH}$ $\rightarrow \mathrm{CH}_{2} \mathrm{~S}+\mathrm{CH}_{2} \mathrm{O}+\mathrm{OH}$ is the main reaction channel.

For these reactions, based on the value of the frequencies, the barrier heights of our ab initio calculation, the total energy $E$ in the microcanonical case and the temperature $T$ in the canonical case, using the RRKM theory, the total amounts of states $W(E)$ and the density of 
states $\rho(E)$ were calculated. The results are shown in Table 2 to 5 . The value of $E$ in the microcanonical case was calculated according to the relationship with $T$ in the canonical case,

$$
E=\sum_{i}\left(\frac{\hbar \omega_{i}}{e^{\hbar \omega_{i} / k T}-1}\right)
$$

Then, the rate constants of the four reactions (R1 to R4) have been calculated in both canonical and microcanonical cases. The results in microcanonical case are listed in Table 2 to 5 , and those results in canonical case are listed in Table 6 to 9. The corresponding data are plotted in Figure 2 to 9. In these figures, the value on the y axis increases as $10^{\mathrm{n}}$. For R1, R2 and R3, they are all the reactions with the same reactant, i.e. $\mathrm{CH}_{3} \mathrm{SCH}_{2} \mathrm{OO}$. However, different results can be observed from Figure 2 to 7 . The rate constants of R1 are plotted in Figures 2 and 3. Specifically, Figures 2 and 3 show the rate constants of R1 in the canonical case and microcanonical case, respectively. In the canonical case, the anharmonic values are larger than the corresponding harmonic values within the whole temperature range (800-3800 K). When $T=700 \mathrm{~K}$, the result we obtained shows that the anharmonic value is $1.53 \times 10^{5} \mathrm{~s}^{-1}$, which is smaller than the corresponding harmonic value $\left(1.62 \times 10^{5} \mathrm{~s}^{-1}\right)$. That means the junction arises when $T$ is between $700 \mathrm{~K}$ and $800 \mathrm{~K}$. In the microcanonical case, when $T=800$ and $1000 \mathrm{~K}$, the energies are lower than the calculated activation energy, i.e. $21.10 \mathrm{kcal} / \mathrm{mol}$. Hence, Table 2 lists the calculated rate constants at those energies which are higher than 
$21.10 \mathrm{kcal} / \mathrm{mol}$. Figure 3 shows that the property of the anharmonic effect is similar to that in canonical case. Figures 2 and 3 also show that the gap between the anharmonic and harmonic values increases with the increasing temperature or total energy. When $T=800,2400$ and $3800 \mathrm{~K}$, the harmonic rate constant is $1.40 \times 10^{5}, 5.32 \times 10^{9}$ and $4.01 \times 10^{10} \mathrm{~s}^{-1}$, respectively, which is $90.3 \%, 9.6 \%$ and $2.8 \%$ smaller than the anharmonic value. Thus, for reaction $\mathrm{R} 1$, the anharmonic effect becomes more and more significant with increasing temperature. And it is same with increasing total energy in microcanonical case. In high-temperature combustion environment, especially $T=2000 \mathrm{~K}$, the anharmonic rate constant is $1.18 \times 10^{10} \mathrm{~s}^{-1}$, which is 6.56 times larger than the harmonic value. It means the anharmonic effect is quite significant in combustion environment. For the reaction R2, according to figures 4 and 5, the junction arises at almost $2200 \mathrm{~K}$. In the canonical case, when the values of temperature are lower than almost $2200 \mathrm{~K}$, the anharmonic values are lower than corresponding harmonic values. When the values of temperature are higher than almost $2200 \mathrm{~K}$, it is opposite. In hightemperature combustion environment, the anharmonic rate constant is close to the harmonic value. In Table 7, it can be seen that the two values are in the same order of magnitude when $T=2000 \mathrm{~K}$. Hence, for the reaction $\mathrm{R} 2$, the anharmonic effect is not obvious in high-temperature combustion environment. In the microcanonical case, within the whole total energy range $(43.33-140.82 \mathrm{kcal} / \mathrm{mol})$, the anharmonic values are all higher than the harmonic values. The anharmonic effect is not so 
significant for R2. For the reaction R3, according to figures 6 and 7, with either canonical or microcanonical approaches, though the harmonic and anharmonic rate constants yield similar results, the difference becomes decreasingly significant at high temperature and energy. And the anharmonic effect in canonical case is not obvious enough. In microcanonical case, when $E=60.81,132.56$ and $197.61 \mathrm{kcal} / \mathrm{mol}$, the harmonic rate constant is $1.77 \times 10^{7}, 2.80 \times 10^{10}$ and $2.06 \times 10^{11} \mathrm{~s}^{-1}$, respectively, which is $19.7 \%, 58.3 \%$ and $90.8 \%$ smaller than the anharmonic value. In short, for reaction R3, the anharmonic effect is more significant when the total energy is relative low in microcanonical case.

As the reaction which follows $\mathrm{R} 1$, in terms of anharmonic effect, R4 shows an analogous characteristic with R1. However, the different result is that the anharmonic values are lower than the corresponding harmonic values in the range of the whole temperature (400-2200 K). It is same within the whole total energy range in the microcanonical case. By calculating the data of rate constants, the anharmonic effect is less obvious than that for reaction R1.

\subsection{The rate constants of subsequent reactions}

In the subsequent reactions $\mathrm{R} 5$ to $\mathrm{R} 8$, the initial reactants are both $\mathrm{CH}_{2} \mathrm{~S}$ and $\mathrm{HO}_{2}$. After our ab initio calculation, their relative energies of all the transition states and products are shown in Figure 10. The energy of the reactant is set to zero $\mathrm{kcal} / \mathrm{mol}$. 
Using RRKM theory, the rate constants of the first two bimolecular reactions (R5 and R6) have been calculated in canonical case. The results are listed in Tables 10 and 11. And the corresponding data are plotted in Figures 11 and 12. According to the figures, for the two reactions, the anharmonic effect is significant enough in the relative wide range of temperatures. It is especially obvious in high temperature range. According to Tables 10 and 11 , at $2000 \mathrm{~K}$, the harmonic rate constant is much larger than the corresponding anharmonic value. That means the anharmonic effect is much obvious in high-temperature combustion.

Finally, the rate constants of last two reactions (R7 and R8) have been calculated in both canonical and microcanonical cases. The two reactions followed by R6 are extremely meaningful because one of the final products is $\mathrm{SO}_{2}$. Table 12 to 15 list the data we acquired. The results of rate constants are plotted in Figure 13 to 16 . For R7, the rate constants increase with the temperature in the canonical case and the total energy in the microcanonical case. But they show different characteristics in different cases. In canonical case, when the temperature is $800 \mathrm{~K}$, the anharmonic value is $7.68 \times 10^{3} \mathrm{~s}^{-1}$, which is $13.2 \%$ smaller than the harmonic value. While the temperature is $5000 \mathrm{~K}$, the anharmonic value is $9.52 \times 10^{11} \mathrm{~s}^{-1}$, which is $79.3 \%$ smaller than the harmonic value. Thus the anharmonic effect of $\mathrm{R} 7$ is clearer in a relative lower temperature range. In high-temperature combustion environment, especially at $2000 \mathrm{~K}$, the anharmonic value is $2.17 \times 10^{9} \mathrm{~s}^{-1}$, which is $22.7 \%$ smaller than the harmonic value. So the anharmonic effect is quite obvious in the case. In 
microcanonical case, the property of the anharmonic effect is similar to that in canonical case. However, it cannot be ignored that the junction arises. When the total energy is larger than $100 \mathrm{kcal} / \mathrm{mol}$, the anharmonic values become larger than the corresponding harmonic values. For R8, as figures 15 and 16 shown, the anharmonic effect is extremely obvious in both canonical and microcanonical cases. Besides, when $T$ ranges from $800 \mathrm{~K}$ to $5000 \mathrm{~K}$ in canonical case, the anharmonic values show the tendency that it rises firstly and then declines. It is possible that the high temperature affects the reactants, thus it reduce the rate constants of R8. It makes the anharmonic effect become more obvious in high temperature range, especially in high-temperature combustion environment. It is similar in microcanonical case.

\section{Conclusions}

In this study, the geometries of the reactants and the transition states in $\mathrm{CH}_{3} \mathrm{SCH}_{2} \mathrm{OO}$ system have been optimized using the Gaussian 09 program. TS1, TS4, TS5, TS6, TS7 and TS8 have been optimized at the MP2/6-311++G ${ }^{* *}$ level. TS2 and TS3 have been optimized at the B3LYP/6-311++ $\mathrm{G}^{* *}$ level. Reactions $\mathrm{R} 1$ and $\mathrm{R} 4$ are two main reactions because of their lower barrier heights. For the reactions except R5 and R6, the total number of transition states and the density of states of the reactants have been calculated using the RRKM theory. Generally speaking, in the study, for most reactions, the rate constants increase with the temperature in the canonical case and the total energy in the 
microcanonical case. As the important parts of the main reaction channel, the two reactions R1 and R4 should be of great concern. For reaction R1 in the microcanonical case, when $\mathrm{E}=60.03$ and $140.82 \mathrm{kcal} / \mathrm{mol}$, the anharmonic rate constant is $3.69 \times 10^{9}$ and $9.35 \times 10^{11} \mathrm{~s}^{-1}$, respectively, which is 4.8 and 26.6 times of the harmonic value. The difference between the two kinds of rate constants is obviously large at higher energy, which means the anharmonic effect is becoming more and more obvious with the increasing energy. For reaction R4 in the microcanonical case, when $\mathrm{E}=9.58$ and $70.65 \mathrm{kcal} / \mathrm{mol}$, the harmonic rate constant is $5.24 \times 10^{10}$ and $8.54 \times 10^{11} \mathrm{~s}^{-1}$, respectively, which is 1.30 and 1.34 times of the anharmonic value. Thus the anharmonic effect is less obvious than that for reaction R1. And the harmonic values are larger than the anharmonic values. It is also different from the reaction R1. The similar phenomenon appears in the canonical case. Besides, the anharmonic effect is various for the other reactions. It is worth noting that the anharmonic effect is extremely obvious for reactions R5, R6, and R8. According to the result shown in Table 1, their barrier heights are relatively low to $5.57,9.73$ and $11.50 \mathrm{kcal} / \mathrm{mol}$, respectively. Therefore we can draw conclusion that the anharmonic effect is relatively obvious for those reactions whose barrier heights are relatively low. By comparing the data in Tables 10,11 and $13(T=2000 \mathrm{~K})$, there is 3, 3 and 1 order of magnitude difference between harmonic rate constant and anharmonic value for the reactions R5, R6 and R8, respectively. Thus we come up with a result that the anharmonic effect is quite obvious for 
those reactions whose barrier heights are relatively low in our research system, especially in high-temperature combustion environment,.

In this paper, the microcanonical and canonical results are generally similar, which indicates that RRKM theory is suitable for the calculation of the rate constant of these reactions. The anharmonic effect of the eight reactions for $\mathrm{CH}_{3} \mathrm{SCH}_{2} \mathrm{OO}$ system cannot be neglected.

\section{Acknowledgments}

This work was supported by the Major Research plan of the National Natural Science Foundation of China (Grant No. 91441132). The Fundamental Research Funds for the Central Universities (Grant Nos. $3132016127,3132016326)$ 


\section{References}

(1) Pirjola, L.; O’Dowd, C. D.; Brooks, I. M.; Kulmala, M. J. Geophys. Res. 2000, 105, 26531-26546.

(2) Berndt, T.; Boge, O.; Stratmann, F.; Heintzenberg, J.; Kulmala, M. Science. 2005, 307, 698-700.

(3) Quinn, P. K.; Bates, T. S. Nature. 2011, 48, 51-56.

(4) Barnes, I.; Hjorth, J.; Mihalopoulos, N. Chem. Rev. 2006, 106, 940-975.

(5) Wu, R. et al. J. Phys. Chem A. 2015, 119 (1), 112-117.

(6) Lucas, D. D.; Prinn, R. G. Atmos. Chem. Phys. 2005, 5, 1505-1525.

(7) Mueller, S. F.; Mao, Q.; Mallard, J. W. Atmos. Chem. Phys. 2011, 11, 290-320.

(8) Yu, F.; Luo, G. Atmosphere 2010, 1, 34-50.

(9) Osthoff, H. D.; Bates, T. S.; Johnson, J. B.; Kuster, W. C.; Goldan, P.; Sommariva, R.; Williams, E. J.; Lerner, B. M.; Warneke, C.; de Gouw, J. A.; et al. J. Geoph ys. Res. 2009, 114, D07301

(10) Boucher, O.; Moulin, C.; Belviso, S.; Aumont, O.; Bopp, L.; Cosme, E.; von Kuhlmann, R.; Lawrence, M. G.; Pham, M.; Reddy, M. S.; et al. Atmos. Chem. Phys. $2003,3,49-65$.

(11) Karl, M.; Gross, A.; Leck, C.; Pirjola, L. J. Geophys. Res. 2007 , 112, D15304.

(12) Faloon, I. Atmos. Environ. 2009, 43, 2841-2854.

(13) Jin, F.; Asatryan, R.; Bozzelli, J. W. Int. J. Quantum Chem. 2012, 112, 1945-1958.

(14) Miller, J. A.; Pilling, M. J.; Troe, J. Proc. Combust. Inst. 2005, 30, 43-88.

(15) H. Eyring. J. Chem. Phys. 3, 107 (1935).

(16) (a) W. Forst.; Z.J. Prasil. Chem. Phys. 53, 3065 (1970); (b) W. Forst. Chem. Rev. 71, 339 (1971); (c) W. Forst. Academic Press. 1973.

(17) M.R. Hoare.; Th.W. Ruijgrok. J. Chem. Phys. 52, 113 (1970).

(18) H. Eyring.; S.H. Lin.; S.M. Lin. Wiley-Interscience. 1980.

(19) (a) L. Yao.; A.M. Mebel.; H.F. Lu.; H.J. Neusser.; S.H. Lin. J. Phys. Chem. A 111, 6727(2007); (b) L. Yao.; S.H. Lin. Mod. Phys. Lett. B 22, 3043 (2008); (c) L. Yao.; S.H. Lin. Sci. China Ser. B 51, 1146 (2008); (d) L. Yao.; R.X. He.; A.M. Mebel.; S.H. Lin. Chem. Phys. Lett. 470, 210 (2009); (e) Ying Shao.; Li Yao.; ShengHsien Lin. Chem. Phys. Lett. 478, 277 (2009); (f) L.Yao.; A.M. Mebel.; S.H. Lin. J. Phys. Chem. A 113, 14664 (2009).

(20) T. Baer.; W.L. Hase. Oxford University Press, 1996.

(21) R.G. Gilbert.; S.C. Smith. Blackwell, 1990.

(22) Baer, T.; Hase, W. L. Theory and experments, 1996. 


\section{Tables and figures}

Table 1. Eight reactions and their barrier heights.

Table 2. The rate constants of R1 at different energies for the microcanonical system. The units of total energy, $\rho(\mathrm{E})$ and rate constants are, respectively, $\mathrm{kcal} / \mathrm{mol}, 1 / \mathrm{cm}^{-1}$ and s $\mathrm{s}^{-1}$.

Table 3. The rate constants of $\mathrm{R} 2$ at different energies for the microcanonical system. The units of total energy, $\rho(\mathrm{E})$ and rate constants are, respectively, $\mathrm{kcal} / \mathrm{mol}, 1 / \mathrm{cm}^{-1}$ and s $\mathrm{s}^{-1}$.

Table 4. The rate constants of R3 at different energies for the microcanonical system. The units of total energy, $\rho(\mathrm{E})$ and rate constants are, respectively, $\mathrm{kcal} / \mathrm{mol}, 1 / \mathrm{cm}^{-1}$ and s $\mathrm{s}^{-1}$.

Table 5. The rate constants of R4 at different energies for the microcanonical system. The units of total energy, $\rho(\mathrm{E})$ and rate constants are, respectively, $\mathrm{kcal} / \mathrm{mol}, 1 / \mathrm{cm}^{-1}$ and $\mathrm{s}^{-1}$.

Table 6 . The rate constants of R1 at different temperatures for the canonical system. The units of temperature, total energy and rate constants are $\mathrm{K}, \mathrm{kcal} / \mathrm{mol}_{\text {and }} \mathrm{s}^{-1}$, respectively.

Table 7. The rate constants of R2 at different temperatures for the canonical system. The units of temperature, total energy and rate constants are $\mathrm{K}, \mathrm{kcal} / \mathrm{mol}$ and s ${ }^{-1}$, respectively.

Table 8. The rate constants of R3 at different temperatures for the canonical system. The units of temperature, total energy and rate constants are $\mathrm{K}, \mathrm{kcal} / \mathrm{mol}$ and s${ }^{-1}$, respectively.

Table 9. The rate constants of R4 at different temperatures for the canonical system. The units of temperature, total energy and rate constants are $\mathrm{K}, \mathrm{kcal} / \mathrm{mol}$ and $\mathrm{s}^{-1}$, respectively.

Table 10. The rate constants of R5 at different temperatures for the canonical system. The units of temperature and rate constants are $\mathrm{K}$ and $\mathrm{cm}^{3} / \mathrm{mol} / \mathrm{s}$, respectively.

Table 11. The rate constants of R6 at different temperatures for the canonical system. The units of temperature and rate constants are $\mathrm{K}$ and $\mathrm{cm}^{3} / \mathrm{mol} / \mathrm{s}$, respectively.

Table 12. The rate constants of R7 at different temperatures for the canonical system. The units of temperature, total energy and rate constants are $\mathrm{K}, \mathrm{kcal} / \mathrm{mol}$ and $\mathrm{s}^{-1}$, respectively.

Table 13. The rate constants of R8 at different temperatures for the canonical system. The units of temperature, total energy and rate constants are $\mathrm{K}, \mathrm{kcal} / \mathrm{mol}$ and $\mathrm{s}^{-1}$, respectively.

Table 14. The rate constants of R7 at different energies for the microcanonical system. The units of total energy, $\rho(E)$ and rate constants are $\mathrm{kcal} / \mathrm{mol}, 1 / \mathrm{cm}^{-1}$ and s ${ }^{-1}$, respectively.

Table 15. The rate constants of R8 at different energies for the microcanonical system. The units of total energy, $\rho(E)$ and rate constants are $\mathrm{kcal} / \mathrm{mol}, 1 / \mathrm{cm}^{-1}$ and s ${ }^{-1}$, respectively. 
Figure 1. The relative energy diagram of the reactions R1 to R4. The unit of energy $\mathrm{E}$ is $\mathrm{kcal} / \mathrm{mol}$. Figure 2. The canonical rate constants of $\mathrm{R} 1$. The unit of rate constant $\mathrm{k}$ is s${ }^{-1}$.

Figure 3. The microcanonical rate constants of R1. The unit of rate constant $\mathrm{k}^{-1} \mathrm{~s}^{-1}$. Figure 4. The canonical rate constants of $\mathrm{R} 2$. The unit of rate constant $\mathrm{k}_{\text {is s }}{ }^{-1}$.

Figure 5. The microcanonical rate constants of $\mathrm{R} 2$. The unit of rate constant $\mathrm{k}^{-1} \mathrm{~s}^{-1}$. Figure 6. The canonical rate constants of R3. The unit of rate constant $\mathrm{k}$ is s${ }^{-1}$.

Figure 7. The microcanonical rate constants of $\mathrm{R} 3$. The unit of rate constant $\mathrm{k}^{-1} \mathrm{~s}^{-1}$. Figure 8. The canonical rate constants of $\mathrm{R} 4$. The unit of rate constant $\mathrm{k}^{-1}$ is s .

Figure 9. The microcanonical rate constants of $\mathrm{R} 4$. The unit of rate constant $\mathrm{k}_{\text {is s }}{ }^{-1}$.

Figure 10. The relative energy diagram of the reactions R5 to R8. The unit of energy E is $\mathrm{kcal} / \mathrm{mol}$.

Figure 11. The canonical rate constants of R5. The unit of rate constant $\mathrm{k}$ is cm $3 / \mathrm{mol} / \mathrm{s}$.

Figure 12. The canonical rate constants of R6. The unit of rate constant $\mathrm{k}$ is cm$/ 3 / \mathrm{mol} / \mathrm{s}$.

Figure 13. The canonical rate constants of R7. The unit of rate constant $\mathrm{k}$ is s${ }^{-1}$.

Figure 14. The microcanonical rate constants of R7. The unit of rate constant $\mathrm{k}^{-1} \mathrm{~s}^{-1}$.

Figure 15. The canonical rate constants of $\mathrm{R} 8$. The unit of rate constant $\mathrm{k} \mathrm{is} \mathrm{s}^{-1}$.

Figure 16. The microcanonical rate constants of $\mathrm{R} 8$. The unit of rate constant $\mathrm{k}$ is $\mathrm{s}^{-1}$. 
Table 1

\begin{tabular}{cccc}
\hline \multirow{2}{*}{ Reactant } & Reaction & Label & $\begin{array}{c}\text { Barrier height } \\
(\mathrm{kcal} / \mathrm{mol})\end{array}$ \\
\hline \multirow{2}{*}{$\mathrm{CH}_{3} \mathrm{SCH}_{2} \mathrm{OO}$} & $\mathrm{CH}_{3} \mathrm{SCH}_{2} \mathrm{OO} \rightarrow \mathrm{TS} 1 \rightarrow \mathrm{CH}_{2} \mathrm{SCH} \mathrm{CH}_{2} \mathrm{OOH}$ & $\mathrm{R} 1$ & 21.10 \\
\cline { 2 - 4 } & $\mathrm{CH}_{3} \mathrm{SCH}_{2} \mathrm{OO} \rightarrow \mathrm{TS} 2 \rightarrow \mathrm{CH}_{3} \mathrm{SC}(=\mathrm{O}) \mathrm{H}+\mathrm{OH}$ & $\mathrm{R} 2$ & 38.80 \\
\cline { 2 - 4 } & $\mathrm{CH}_{3} \mathrm{SCH}_{2} \mathrm{OO} \rightarrow \mathrm{TS} 3 \rightarrow \mathrm{CH}_{3} \mathrm{~S}(=\mathrm{O})+\mathrm{CH}_{2} \mathrm{O}$ & $\mathrm{R} 3$ & 40.12 \\
\hline $\mathrm{CH}_{2} \mathrm{SCH}_{2} \mathrm{OOH}$ & $\mathrm{CH}_{2} \mathrm{SCH}_{2} \mathrm{OOH} \rightarrow \mathrm{TS} 4 \rightarrow \mathrm{CH}_{2} \mathrm{~S}+\mathrm{CH}_{2} \mathrm{O}+\mathrm{OH}$ & $\mathrm{R} 4$ & 4.03 \\
\hline \multirow{2}{*}{$\mathrm{CH}_{2} \mathrm{~S}+\mathrm{HO}_{2}$} & $\mathrm{CH}_{2} \mathrm{~S}+\mathrm{HO}_{2} \rightarrow \mathrm{TS} 5 \rightarrow \mathrm{HOOCH}_{2} \mathrm{~S}$ & $\mathrm{R} 5$ & 5.57 \\
\hline & $\mathrm{CH}_{2} \mathrm{~S}+\mathrm{HO}_{2} \rightarrow \mathrm{TS} 6 \rightarrow \mathrm{CH}_{3} \mathrm{SOO}$ & $\mathrm{R} 6$ & 9.73 \\
\hline $\mathrm{CH}_{3} \mathrm{SOO}$ & $\mathrm{CH}_{3} \mathrm{SOO} \rightarrow \mathrm{TS} 7 \rightarrow \mathrm{CH}_{3} \mathrm{SO}_{2}$ & $\mathrm{R} 7$ & 30.99 \\
\hline $\mathrm{CH}_{3} \mathrm{SO}$ & $\mathrm{CH}_{3} \mathrm{SO}_{2} \rightarrow \mathrm{TS} 8 \rightarrow \mathrm{CH}_{3}+\mathrm{SO}_{2}$ & $\mathrm{R} 8$ & 11.50 \\
\hline
\end{tabular}

